\title{
A Model for the Break-up of a Tuft of Fibers
}

\author{
Michael E. M. Lee \\ BP Institute for Multiphase Flow, University of Cambridge, Madingley Road, Cambridge, CB3 OEZ, UK \\ Gregory Kozyreff \\ Optique Nonlinéaire Théorique, Université Libre De Bruxelles, \\ C. P. 231, Campus de la Plaine, B-1050 Bruxelles, Belgium \\ Peter D. Howell and Hilary Ockendon \\ OCIAM, Mathematical Institute, 24-29 St. Giles', Oxford OX1 3LB, UK \\ (Dated: August 20, 2006)
}

\begin{abstract}
A simple model for the forces acting on a single fiber as it is withdrawn from a tangled fiber assembly is proposed. Particular emphasis is placed on understanding the dynamics of the reptating fiber with respect to the entanglement of fibers within the tuft. The resulting two-parameter model captures the qualitative features of experimental simulation. The model is extended to describe the break-up of a tuft. The results show good agreement with experiment and indicate where a tuft is most likely to fracture based on the density of fiber end-points.
\end{abstract}

PACS numbers: $62.40 .+1,46.55 .+\mathrm{d}, 46.70 .-\mathrm{p}$

Keywords: tuft, textiles, reptation, fiber assembly

\section{MOTIVATION}

There has been considerable interest in understanding the physical properties of media that are partly composed of fibers or nematic objects; examples are fiber suspensions [1-3], fiber reinforced composites $[4,5]$, polymers [6] and liquid crystals [7]. For textile fibers within dense tufts, their relative orientations within an assembly and the number of frictional contact points govern the internal forces of the tuft. In compression and for small deformations in extension the bending of individual fibers allows the whole assembly to distort without significant slippage between fibers and an anisotropic elastic model can be used [8]. However under higher extensional loads, the fibers must slip over each other causing an irreversible deformation and it is this type of distortion that we are concerned with here. Modelling these interactions, in particular aggregate or bulk behaviour, is not well understood but there has been some recent work. For example $[9,10]$ present models for extensional deformation that are based on planar constrained fiber mechanics and $[11,12]$ consider fiber withdrawal from an ad hoc point of view. More recently [13] has studied the dynamics of fiber slippage under small cyclic loads. A different approach was taken in [14], which derives a 3D phenomenological continuum model that includes density, alignment, order and entanglement as dependent variables.

The original aim of this work was to understand and quantify the forces acting inside a tuft of fibers as it is processed by the carding machine, but the theory developed here applies to any mass of long tangled fibers which are subject to extensional forces. As a tuft goes through the carding machine it is subjected to a series of forces aimed at teasing, separating and aligning the fibers so that an ordered lap is obtained. Typically a tuft is torn apart either under tension or under shearing forces, and the forces resisting the deformation of the tuft will be those acting between neighbouring fibers in the tuft; see [15] for a recent experimental study.

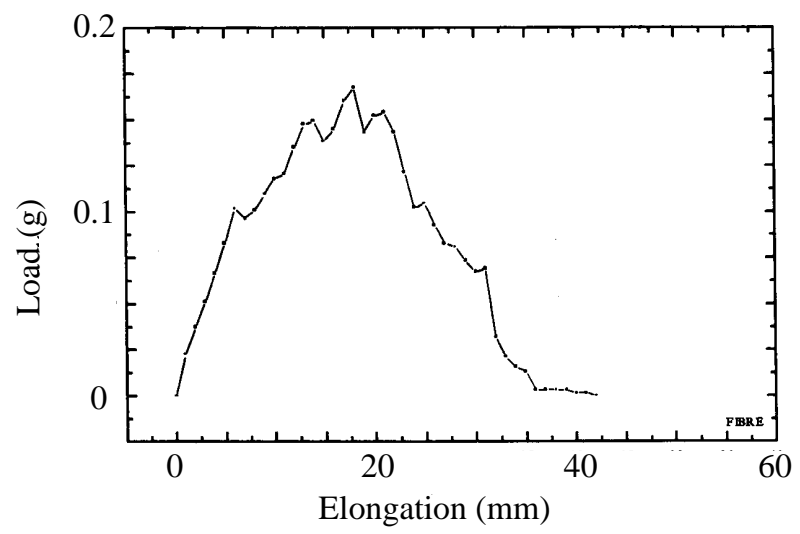

FIG. 1: Experimental results of a single fiber being withdrawn from a tuft at $50 \mathrm{~mm} / \mathrm{min}$; polyester fibers $1.5 \mathrm{dtex}$.

In order to aid the creation of a model for the deforming tuft a series of experiments were set up in order to elucidate the dependence of the forces on the parameters of the problem. The experimental work was conducted by Dr Mahmoudi at the Centre for Technical Textiles, University of Leeds, and some of this work has been published in [16]. In the first experiment, a single fiber was removed at constant speed from a clamped tuft and the tension in the fiber was recorded as shown in Figure 1. The graph illustrates the results of a large number of experiments which have been normalised to allow for variation in the size of the initial tuft. In a second experiment a tuft was clamped at both ends which were then pulled apart with constant speed and the force experienced was 
recorded as shown in Figure 2. Again, this experiment was performed many times and repeated for a number of different fiber types and tuft sizes.

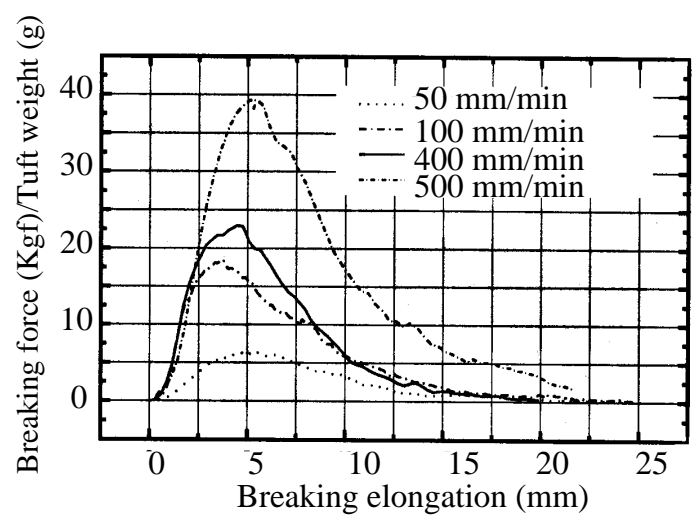

FIG. 2: Graphs of the tuft breaking force experiment for cotton with variable elongation velocities; cotton fibers.

In the first part of this paper we model the first experiment by assuming that the tuft is a fixed uniform structure from which a single fiber is withdrawn. We propose two different friction laws, first a friction which just depends on the number of contact points between the fibers which might be true for rough fibers such as wool. Secondly we assume that the friction depends linearly on the velocity difference between the fibers at a contact point. Both these laws are simpler than the one proposed by [8] and we are thus able to present relatively simple solutions whilst still capturing the essential dynamics. Comparison with experiments allows us to determine the crucial parameters in each case and to assess the value of the model. The second part of the paper uses the results for a single fiber to make a model for the break-up of a tuft. These results are compared with those of the second experiment, in Figure 2, and conclusions about the two models are drawn.

\section{MODELLING PRELIMINARIES}

We first consider a singe fiber which is withdrawn at constant speed $U$ from a fixed tuft. The tuft is assumed to consist of randomly oriented fibers with a uniform volume fraction and we suppose that initially the moving fiber is loosely entangled within the tuft. The fiber will be crimped but we assume that it is inextensible. As the fiber is withdrawn, the part of the fiber outside the tuft will straighten, and the rest of the fiber will come into frictional contact with fibers in the tuft. As the fiber moves out of the tuft it will come into active contact with more and more of the tuft fibers until the whole fiber is in motion. During this part of the motion the tension in the fiber will increase as more frictional contact points are activated. Thereafter, as the fiber continues to move out of the tuft, the number of contact points will decrease, and so will the tension, until the fiber is completely withdrawn from the tuft. Throughout we assume the motion of the other fibers within the tuft is negligible.

\section{A. Effective length of the fiber}

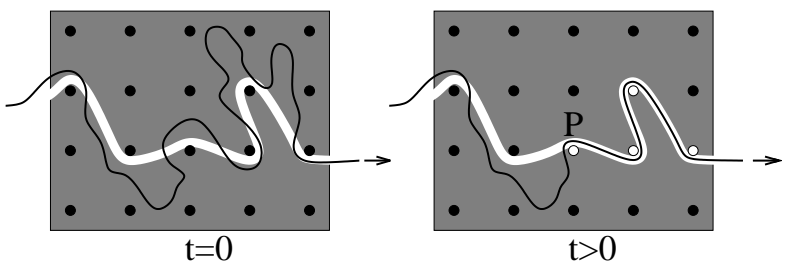

FIG. 3: Schematic illustration of a fiber being removed from a tuft. The unshaded line indicates the shortest trajectory for the fiber's withdrawal when constrained by neighbouring fibers represented here by dots.

The effective length of a fiber is defined as the length over which the friction forces act. As described above this will first increase and then decrease and the process is illustrated schematically in Figure 3. Here the black dots represent the fixed fibers within the tuft and we can see how the length of moving fiber which is in contact with these neighbouring fibers increases as the moving fiber is withdrawn from the tuft. Once contact has been made, the moving fiber will be restricted to moving along the unshaded path, the shortest withdrawal trajectory, in a way that is analogous to reptation in polymers, see [6]. The motion of the fiber is thus constrained by the topological properties of the tuft. For highly tangled, dense textile tufts, we assume that the neighbouring points of the tuft array are stationary, and that the stresses and forces induced by the motion of the single fiber do not affect the structure of the tuft. A similar idea has been used by [13] to model the withdrawal of a fiber from an assembly to describe "pilling" in knitted and woven materials.

The effective length of the fiber that lies along the unshaded path in Figure 3 will increase at a rate which depends on the "state of entanglement" of the tuft. This state of entanglement needs to take account of two different properties of the fiber mass; the first of these is the "slackness" of a fiber within the tuft and the second is the "tortuosity" of the final fiber path around the fixed fibers in the tuft. These ideas have also been discussed by [17] who propose a diffusion model to take account of both effects.

In our simple model we measure the initial slackness $S$ as the ratio of the fiber length to the shortest withdrawal trajectory as shown in Figure 3. Once the fiber is in motion under tension, the displacement of the fiber will converge onto the shortest withdrawal trajectory. The rate at which the fiber slackness between contact points 
is reduced will determine the rate at which the effective length increases since it is not until the slackness between initial contact points is eliminated that further contact points can be engaged. To see the connection between these two rates, we consider a fiber which is moving with speed $U$ through a fixed fiber structure. If the average distance apart between contact points on any particular fiber is $d$ and the slackness parameter is $S$ then we can assume that between two adjacent contact points there is initially a loop of fiber of length $S d$. Thus the fiber must travel a distance $S d-d$ in order to pick up a new contact point and this will take time $(S-1) d / U$. In the same time the effective length will increase by $d$ and so the last contact point ( $\mathrm{P}$ in Figure 3 ) will move with speed $U /(S-1)$ through the tuft in a direction opposite to $U$. Thus the contact point will travel along the fiber with speed $-U(1+1 /(S-1))=-\alpha U$ where $\alpha=S /(S-1)$. The parameter $\alpha$ is always greater than unity and an alternative way to define $\alpha$ is to note that if the fiber is of length $L$ then, ignoring elasticity, $L / \alpha$ is the length of the fiber that has been withdrawn at the time when the far end of the fiber first starts to move. We assume that $\alpha$ is the same constant for all the experiments considered here; if we are considering a tuft of fibers just before it enters the carding machine, this assumption is reasonable for a given material but it is not so reasonable to assume that $\alpha$ will not vary from one material to another.

To parameterize the "tortuosity" of a fiber, we just count the number of contact points per unit length, $\nu$, along the shortest withdrawal trajectory. Clearly the frictional forces per unit length on a fiber will be directly proportional to $\nu$ but it will also depend on the angle that the moving fiber turns through at each contact point. This has been modelled using the Capstan effect, see [17], but in this paper these effects are all contained within the average frictional force per unit length $\hat{f}$. We also assume that the tuft is isotropic throughout this paper.

\section{B. Fiber-fiber interactions}

The forces acting on the moving fiber are the frictional forces due to fiber-fiber interactions. These forces act over the effective length and are proportional to the number of contact points per unit length. We will consider two different friction laws:

1. a constant friction law which assumes that the friction at each moving contact point is a given constant and

2. a dynamic friction law which assumes that the friction generated between two fibers is proportional to the relative velocity of the fibers.

\section{Elastic Effects}

Although the fibers are virtually inextensible the crimp in a fiber has an effect similar to elasticity. It can be shown, [18], that the extension of a crimped fiber depends on its tension as shown in Figure 4. The value of $T_{1}$ where the extension has reached $95 \%$ of the final extension is $\mathcal{O}\left(E I a d^{-2}\right)$ where $E I$ is the bending stiffness of the fibers, $a$ is the amplitude of the crimp and $d$ is the mean wavelength. The breaking tension of the fiber, $T_{2}$, is usually large compared to $T_{1}$.

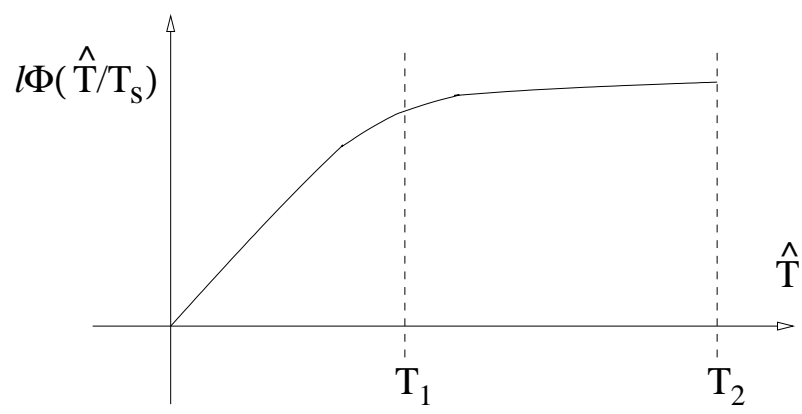

FIG. 4: Extension plotted against tension for a crimped fiber.

The maximum tension experienced in the experimental work (Figures 1,2) is also large compared to $T_{1}$ so it can be assumed that the elastic effect of crimping is only important at the very beginning of the fiber withdrawal experiment.

\section{FIBER WITHDRAWAL PROBLEM}

To derive the equations of motion of the fiber we use Lagrangian variables so that the ends of the fiber are at $\hat{\xi}=0$ and $\hat{\xi}=\ell$. As shown schematically in Figure 5, the fiber has three sections.

1. $0<\hat{\xi}<\hat{\xi}_{1}(t)$ : the fiber is loosely entangled within the tuft and is at rest

2. $\hat{\xi}_{1}(t)<\hat{\xi}<\hat{\xi}_{2}(t)$ : the fiber is moving in contact with stationary fibers within the tuft

3. $\hat{\xi}_{2}(t)<\hat{\xi}<\ell$ : the fiber has been withdrawn from the tuft.

The actual position of a point of the moving fiber is $y(\hat{\xi}, t)$ where $y$ is measured from the point 0 where the fiber leaves the tuft and is measured along the line of the taut part of the fiber within the tuft. Thus

$$
y(\ell, t)=U t, \quad y\left(\hat{\xi}_{2}, t\right)=0 \quad \text { and } \quad \frac{d \hat{\xi}_{1}}{d t}=-\alpha \frac{\partial y}{\partial t}\left(\hat{\xi}_{1}, t\right)
$$

From a simple force balance on an element $\delta \hat{\xi}$ of a fiber under tension $\hat{T}(\hat{\xi})$, the equation of motion of the moving 


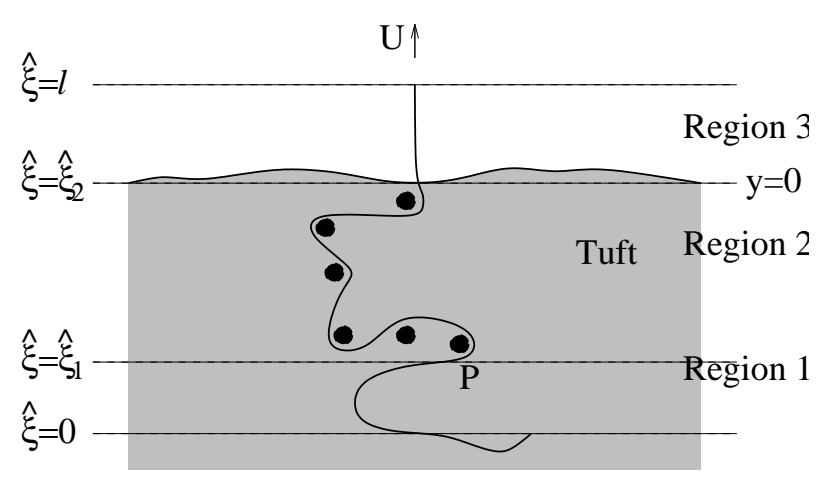

FIG. 5: A schematic illustration of a fiber as it is withdrawn from a tuft.

fiber is

$$
\sigma \frac{\partial^{2} y}{\partial t^{2}}=\frac{\partial \hat{T}}{\partial \hat{\xi}}-\hat{f} \frac{\partial y}{\partial \hat{\xi}}
$$

where $\hat{f}$ is the mean force per unit length opposing the motion of the fiber and $\sigma$ is the density per unit length of the fiber in the unextended state. The term $\partial y / \partial \hat{\xi}$ in (2) is the local extension ratio between the deformed element $\delta y$ and the same, undeformed element of fiber $\delta \hat{\xi}$. The elasticity law is

$$
\frac{\partial y}{\partial \hat{\xi}}=1+\Phi\left(\frac{\hat{T}}{T_{s}}\right)
$$

where $\Phi$ is the modulus sketched in Figure 4 and $T_{s}$ is a typical value of the tension. In region $3, \hat{f}=0$ but in region 2 , the force $\hat{f}$ will be the mean friction per unit length described in section II B.

We non-dimensionalise the variables by writing

$$
\begin{aligned}
y & =\ell Y, \quad t=\frac{\ell}{U} \tau, \quad \hat{T}=T_{s} T, \quad \hat{f}=\frac{T_{s}}{\ell} f, \\
\hat{\xi}_{i} & =\ell \xi_{i} \quad \text { and } \quad \hat{\xi}=\ell \xi,
\end{aligned}
$$

where $T_{s}$ is still to be chosen. It turns out that in all cases the parameter $\frac{\sigma U^{2}}{T_{s}}$ is small and so henceforth we neglect the left hand side of equation (2). Thus we need to solve the quasi-static model which, in non-dimensional terms, is

$$
\frac{\partial T}{\partial \xi}=f \frac{\partial Y}{\partial \xi}, \quad \frac{\partial Y}{\partial \xi}=1+\Phi(T) .
$$

Solving these equations (5) with boundary conditions from (1), in region 3 where $f=0$ we find that

$$
T=F(\tau) \quad \text { and } \quad Y=(1+\Phi(F))(\xi-1)+\tau
$$

where $(1+\Phi(F))\left(\xi_{2}-1\right)+\tau=0$. The boundary conditions for region 2 will therefore be

$$
\begin{aligned}
T & =F(\tau) \text { and } Y=0 \quad \text { at } \xi=\xi_{2}, \\
\text { and } T & =0, \quad \frac{d \xi_{1}}{d \tau}=-\alpha \frac{\partial Y}{\partial \tau} \quad \text { at } \xi=\xi_{1}
\end{aligned}
$$

where the second boundary condition (8) holds as long as $\xi_{1}>0$ but is replaced by

$$
T=0 \text { at } \xi=0
$$

once $\xi_{1}$ has reached zero and the whole fiber is in motion. In addition, we need to add the initial conditions

$$
\xi_{1}(0)=1, \quad \xi_{2}(0)=1 .
$$

For the moment, we ignore the elastic effects by taking $\Phi(T)=0$ and the problem can then be solved analytically. It is easy to see that

$$
Y=\xi-\xi_{2}, \quad \xi_{2}=1-\tau, \quad \xi_{1}=1-\alpha \tau
$$

for $0<\tau<\frac{1}{\alpha}$. To determine $F(\tau)$, we need to look at the two possible friction laws separately.

\section{A. Constant friction law}

In this case we can choose $T_{s}=\hat{f} l$ so that $f=1$ and then

$$
F=\left\{\begin{array}{cc}
\tau(\alpha-1) & \text { if } 0<\tau<\frac{1}{\alpha} \\
1-\tau & \text { if } \frac{1}{\alpha}<\tau<1
\end{array},\right.
$$

as illustrated in figure 6 . Even with this simple friction law, the model (12) captures the essential qualitative features exhibited in the experimental results shown in Figure 1.

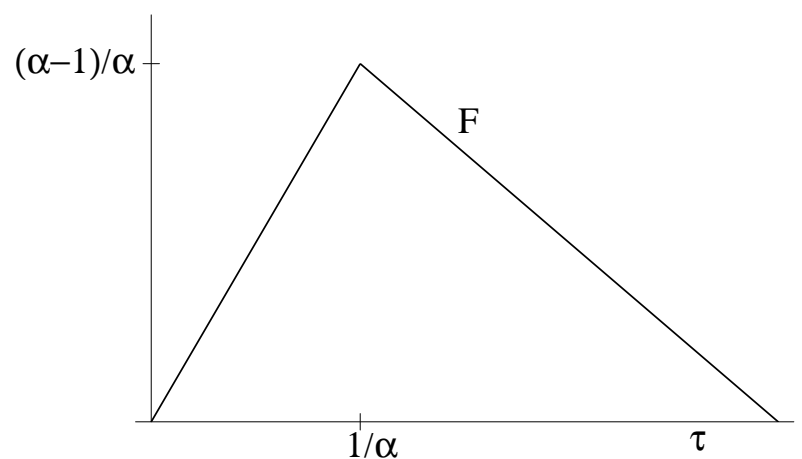

FIG. 6: An illustration for the force given in (12) for the withdrawal of a fiber from a tuft; $\alpha=3$.

\section{B. Dynamic friction law}

If the friction is linearly dependent on the relative velocity of the fibers, then

$$
\hat{f}=\lambda \frac{\partial y}{\partial t}
$$

where $\lambda$ is constant. Non-dimensionalising gives

$$
f=\frac{\partial Y}{\partial \tau}
$$

if we choose $T_{s}=U \lambda \ell$ and so, when $\Phi=0, f=1$ again and the result (12) still holds. 


\section{Experimental Results}

The experimental results shown in Figure 1 were obtained by a series of experiments in which a single polyester fiber was removed at constant speed $U=$ $50 \mathrm{~mm} \mathrm{~min}^{-1}$ from a clamped tuft. In each case the force was measured as a function of distance travelled and the results were drawn on a single graph. Since polyester fiber was used it is reasonable to assume that all fibers have approximately the same length of $40 \mathrm{~mm}$. Comparing these results with the theoretical model allows us to estimate the maximum value of the applied force, $T_{s}(1-1 / \alpha)$, and the time, $L /(U \alpha)$, or distance, $L / \alpha$, at which this maximum occurs. This information is sufficient to determine $T_{s}$ and $\alpha$ but does not enable us to distinguish between the two friction laws proposed. The values given are $\alpha=2$ and $T_{s}=0.33 \mathrm{~g} \mathrm{~m} \mathrm{~s}^{-2}$. Note that the parameter $\sigma U^{2} / T_{s}=\mathcal{O}\left(10^{-13}\right)$ thus justifying our neglect of inertia in equation (5).

\section{Numerical Results}

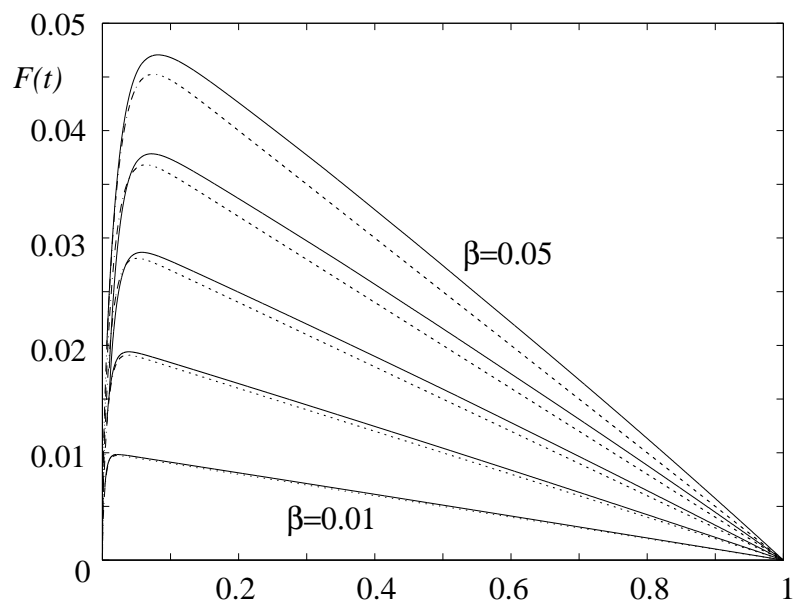

FIG. 7: The withdrawal force on of a single fiber: dotted lines plot asymptotic solutions and the solid lines plot the numerical computations with $\beta=0.01,0.02,0.03,0.04,0.05$ in ascending order for both sets of results and $\alpha=\infty$.

If $\Phi$ varies with $\hat{T}$ as shown in Figure 4 , then it is necessary to resort to numerical methods. The two free boundaries at $\xi=\xi_{1}$ and $\xi=\xi_{2}$ make this a non-trivial exercise; however as long as $T_{1} \ll T_{s}$, the effect will only be to slightly extend the time spent increasing $F$ and to round off the discontinuous slopes in Figure 6 and so we have not performed extensive numerical simulations. The case of the velocity-dependent friction law with $\Phi(T)=\beta T$ and $1 / \alpha=0$ has been calculated and the results are shown in Figure 7 , for a range of values of $\beta$. The dotted lines are asymptotic solutions for small $\beta$ obtained by matching a regular perturbation for
$\tau=\mathcal{O}(1)$ with a boundary layer of $\operatorname{size} \mathcal{O}(\beta)$ at $\tau=0$, see [19]. Note that the effects of elasticity are not significant until $\beta \rightarrow 1$ and that the withdrawal force is monotonically decreasing except for a short initial time period of $\mathcal{O}(\beta)$ when $\beta \ll 1$.

\section{A FIBER BETWEEN TWO TUFTS}

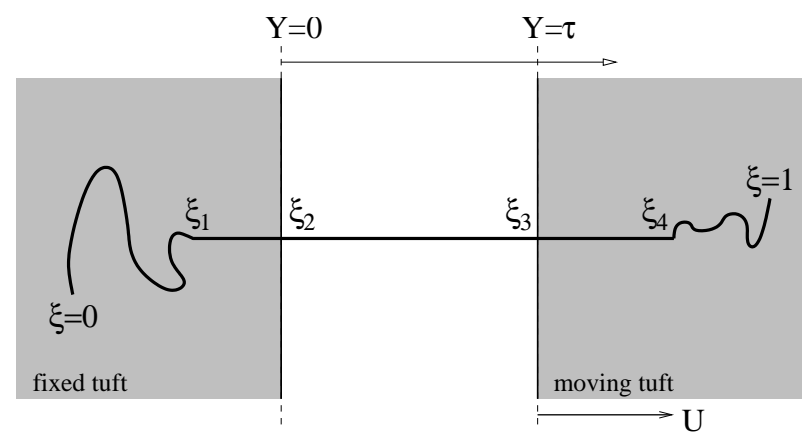

FIG. 8: A schematic diagram of a fiber embedded in two tufts where one tuft moves away from the other tuft at uniform velocity.

Now that the simple experiment has been modelled and yielded the crucial parameters $\alpha$ and $T_{s}$, we can start to build up a model for tuft breakage. We start by analysing the case in which two tufts, connected by a single fiber, are pulled apart at a constant speed $U$. As before, the assumption is that the tufts are undisturbed by the passage of the fiber and we will ignore the elasticity of the fiber and take $\Phi=0$ throughout.

The set-up soon after the start of the motion is illustrated schematically in Figure 8. We assume that initially a length $\theta \ell$ of the fiber is within the fixed lefthand tuft and the rest of the fiber, of length $(1-\theta) \ell$, is initially within the right-hand tuft which moves with constant speed $U$ for $t>0$. The fiber now has five sections which need to be considered separately. We define $\xi_{1}, \xi_{2}, \xi_{3}, \xi_{4}$ as shown in Figure 8 so that at $t=0, \xi_{1}=\xi_{2}=\xi_{3}=\xi_{4}=\theta$. Using the same nondimensionalisation as in section III, we then get the following solution. For $\xi_{2}<\xi<\xi_{3}$, there will be no friction force acting and so

$$
T=F(\tau) \quad \text { and } \quad Y=\xi-\xi_{2} \text { where } \xi_{3}-\xi_{2}=\tau .
$$

For the portions of the fiber which are moving relative to the tufts, $\xi_{1}<\xi<\xi_{2}$ and $\xi_{3}<\xi<\xi_{4}$, we will need to consider the two friction laws separately and this time we find that the results differ in the two cases. 


\section{A. Constant friction law}

In the fixed tuft, with $f=1$, equations (5), (7) and (8) lead to

$$
\begin{aligned}
F(\tau) & =\left\{\begin{array}{cc}
\xi_{2}-\xi_{1} & \text { if } \xi_{1}>0 \\
\xi_{2} & \text { if } \xi_{1}=0
\end{array}\right. \\
\text { where } \xi_{1}-\theta & =\alpha\left(\xi_{2}-\theta\right) \text { when } \xi_{1}>0 .
\end{aligned}
$$

In the moving tuft where $\xi_{3}<\xi<\xi_{4}$ the force $\hat{f}$ will act in the opposite direction so that (5), (7) and (8) are replaced by

$$
\frac{\partial T}{\partial \xi}=-1, \quad T=F(\tau), \quad Y=\tau \quad \text { at } \quad \xi=\xi_{3}
$$

and if $\xi_{4}<1$

$$
T=0, \quad \frac{d \xi_{4}}{d \tau}=\alpha\left(1-\frac{\partial Y}{\partial \tau}\right) \quad \text { at } \quad \xi=\xi_{4},
$$

until $\xi_{4}=1$ then

$$
T=0 \quad \text { at } \quad \xi=1 .
$$

Hence, using (15), we get

$$
F(\tau)=\left\{\begin{array}{cc}
\xi_{4}-\xi_{3} & \text { if } \xi_{4}<1 \\
1-\xi_{3} & \text { if } \xi_{4}=1
\end{array}\right.
$$

where

$$
\xi_{4}-\theta=\alpha\left(\xi_{3}-\theta\right) .
$$

In the first instance $\xi_{1}>0$ and $\xi_{4}<1$ and equations (15), (16), (17), (21) and (22) lead to

$$
\begin{aligned}
& \xi_{1}=\theta-\frac{\alpha \tau}{2}, \quad \xi_{2}=\theta-\frac{\tau}{2}, \quad \xi_{3}=\theta+\frac{\tau}{2}, \\
& \xi_{4}=\theta+\frac{\alpha \tau}{2} \quad \text { and } \quad F(\tau)=(\alpha-1) \frac{\tau}{2} .
\end{aligned}
$$

Without loss of generality, we can assume $0<\theta<\frac{1}{2}$ and so $\xi_{1}$ will become zero before $\xi_{4}=1$ and this will happen when $\tau=\frac{2 \theta}{\alpha}$. Thereafter we need to use $F(\tau)=\xi_{2}$ for $0<\xi<\xi_{2}$ until either $\xi_{4}$ reaches the value of 1 or $\xi_{2}=0$.

However with this simple function law we must remember that it is also possible for the taut part of the fiber to be stationary in a tuft as long as $|f| \leq 1$. Since the length of taut fiber, $\xi_{4}-\xi_{3}$, in the moving tuft cannot decrease until $\xi_{4}=1$ and the length of the fiber in the fixed tuft can only decrease once $\xi_{1}=0$, the only way to balance the forces on the fiber is by keeping $\xi_{3}$ and $\xi_{4}$ fixed while the fiber is extracted from the fixed tuft. Hence, for $\tau>\frac{2 \theta}{\alpha}$,

$$
\begin{aligned}
& \xi_{1}=0, \quad \xi_{2}=\theta\left(1+\frac{1}{\alpha}\right)-\tau, \quad \xi_{3}=\theta\left(1+\frac{1}{\alpha}\right), \\
& \xi_{4}=2 \theta \text { and } F=\theta\left(1+\frac{1}{\alpha}\right)-\tau
\end{aligned}
$$

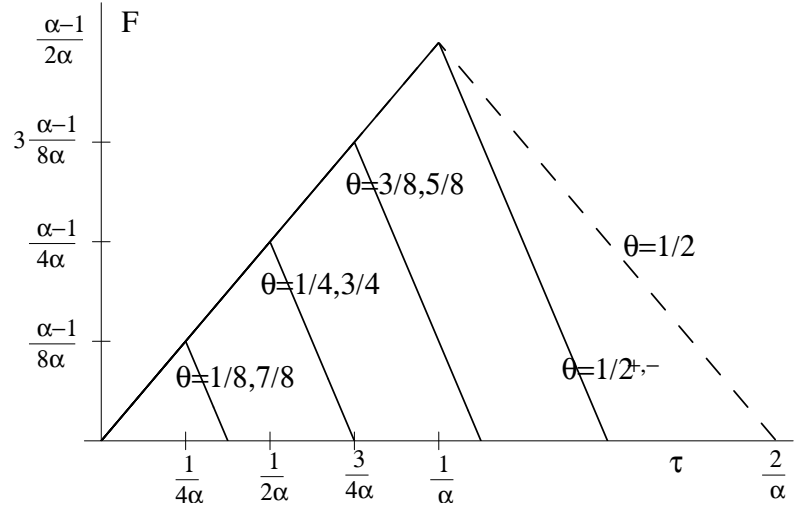

FIG. 9: $F(\tau)$ is defined by (23) and (24), for a range of values of $\theta$ when $\alpha=2$.

and this will persist until $\tau=\theta\left(1+\frac{1}{\alpha}\right)$. When the fiber has been extracted from the fixed tuft, this leaves a "loose end" of length $\theta\left(1+\frac{1}{\alpha}\right)$.

Note that if $\theta=\frac{1}{2}$, symmetry tells us that the fiber will pull out from each tuft at the same rate and so $F=\frac{1}{2}-\frac{\tau}{2}$ when $\tau>1 / \alpha$ and the fiber will detach from both tufts at $\tau=1$. This, however, is an unstable situation since, as soon as $\theta$ is either greater or less than $\frac{1}{2}$, the fiber will remain stationary relative to one of the tufts and $F$ will decrease twice as fast as shown in Figure 9.

\section{B. Dynamic friction law}

Now $f=\frac{\partial Y}{\partial \tau}$ for the taut part of the fiber in the fixed tuft and $f=-\left(1-\frac{\partial Y}{\partial \tau}\right)$ in the moving tuft. Equations (16) and (21) are replaced by

$$
F(\tau)=\left\{\begin{array}{cc}
-\dot{\xi}_{2}\left(\xi_{2}-\xi_{1}\right) & \text { if } \xi_{1}>0 \\
-\dot{\xi}_{2} \xi_{2} & \text { if } \xi_{1}=0
\end{array}\right.
$$

and

$$
F(\tau)=\left\{\begin{array}{cc}
-\dot{\xi}_{3}\left(\xi_{3}-\xi_{4}\right) & \text { if } \xi_{4}<1 \\
-\dot{\xi}_{3}\left(\xi_{3}-1\right) & \text { if } \xi_{4}=1
\end{array}\right.
$$

but relations (17) and (22) still hold. Hence, when $\xi_{1}>0$ and $\xi_{4}<1$, we get the same solution (23) for $\xi_{i}$ but now

$$
F(\tau)=\frac{1}{4}(\alpha-1) \tau \quad \text { for } \quad 0<\tau<\frac{2 \theta}{\alpha} .
$$

In the next part of the motion, $\xi_{1}=0$ but now the fiber continues to slip out of both tufts simultaneously. Assuming that $\dot{\xi}_{3}$ and $\dot{\xi}_{4}$ are both positive and using $\xi_{1}=0$ in (25) gives

$$
\xi_{2}=\frac{1}{\alpha}[(\alpha-1)(\theta-\tau)+\sqrt{(\alpha-1) \tau(2 \theta-\tau)}]
$$

for $\tau>\frac{2 \theta}{\alpha}$ where $\xi_{3}, \xi_{4}$ and $F$ are determined by (15), (22) and $(25)$. 

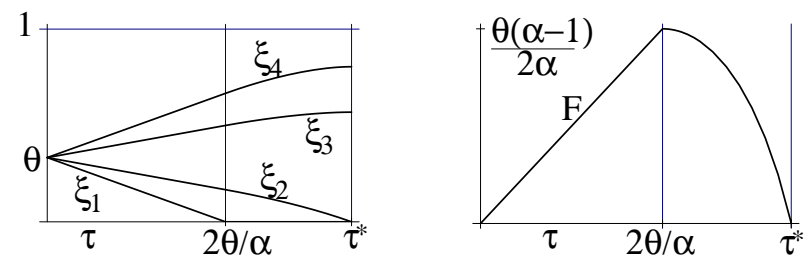

FIG. 10: Graphs of $\xi_{i}$ and the force $F(\tau)$ when $\alpha=2$ and $\theta=\frac{1}{3} ;$ where $\tau^{*}=\frac{\theta(1+\sqrt{\alpha})}{\alpha}$.

It can easily be checked that $\dot{\xi}_{3}>0$ and so this motion persists until either $\xi_{2}=0$ or $\xi_{4}=1$. If $\theta<\frac{1}{1+\sqrt{\alpha}}, \xi_{2}=0$ before $\xi_{4}=1$ and the solution described above continues until $\tau=\theta\left(1-\frac{1}{\sqrt{\alpha}}\right)$ when the fiber is detached from the fixed tuft. The variation of $\xi_{i}$ and $F$ with $\tau$ in this case is illustrated in Figure 10.

If, however, $\frac{1}{1+\sqrt{\alpha}}<\theta<\frac{1}{2}$, then the fiber becomes fully extended inside both tufts when $\xi_{4}=1$ and $\tau=$ $\tau_{1}=\frac{1}{\alpha}+\theta\left(1-\frac{1}{\alpha}\right)-\frac{1}{\alpha} \sqrt{(\alpha-1)\left(\alpha \theta^{2}-(\theta-1)^{2}\right)}$ and this happens before the fiber leaves the fixed tuft.
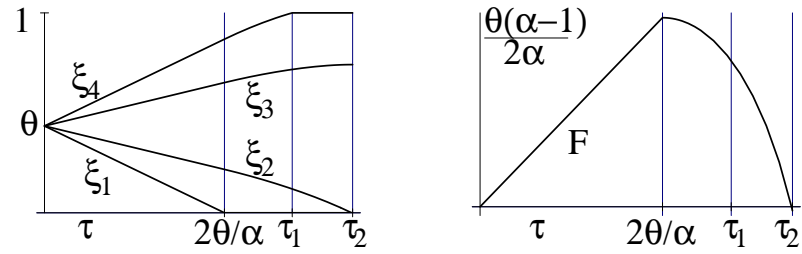

FIG. 11: Graphs of $\xi_{i}$ and the force $F(\tau)$ when $\alpha=2$ and $\theta=\frac{1}{1+\sqrt{2}}+\frac{1}{50}$.

Now there will be a third scenario to consider where $\xi_{1}=0$ and $\xi_{4}=1$. The result in using (25) and (26) is

$$
\begin{aligned}
\xi_{2} & =\frac{1}{2(1-\tau)}\left[(1-\tau)^{2}+\frac{1}{\alpha}(\alpha-1)(2 \theta-1)\right], \\
\xi_{3} & =\xi_{2}+\tau \\
F & =\frac{-1}{4(1-\tau)^{3}}\left[\left(\frac{(\alpha-1)(2 \theta-1)}{\alpha}\right)^{2}-(1-\tau)^{4}\right]
\end{aligned}
$$

and this will be valid from $\tau=\tau_{1}$ until $\tau=\tau_{2}=1-$ $\sqrt{\frac{(\alpha-1)(1-2 \theta)}{\alpha}}$ when $\xi_{2}=0$. The solution is illustrated in Figure 11 .

\section{TUFT BREAKAGE}

It is now possible to construct a simple model for tuft breakage by simulating two tufts connected by a large number $N$ of fibers; the fracture should occur at the structurally weakest region of the tuft. Suppose that the two tufts are touching initially and that a connecting

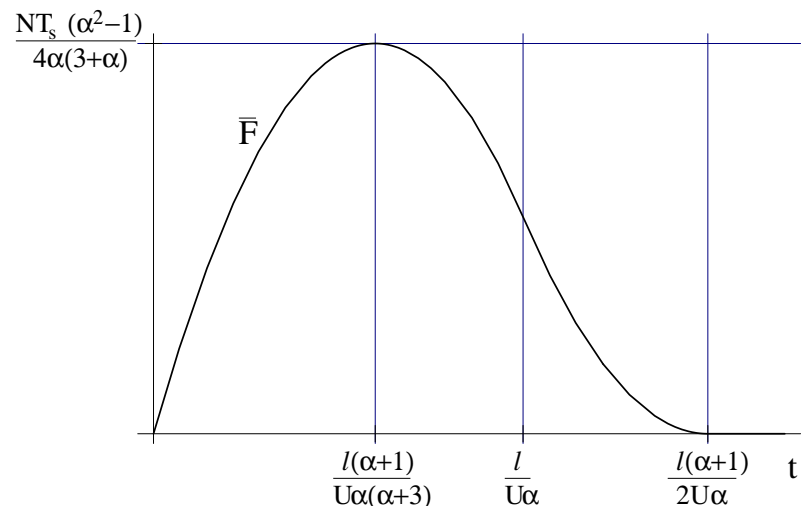

FIG. 12: A plot of the force $\bar{F}$ from (33) for the constant friction law; $\alpha=2$.

fiber has a length $\theta \ell$ in one tuft and $(1-\theta) \ell$ in the other where $\theta$ is distributed on $(0,1)$ with probability density function $p(\theta)$. Then the force needed to pull the tufts apart at speed $U$ will be

$$
\bar{F}(t)=T_{s} N \int_{0}^{1} F(\theta, U t / \ell) p(\theta) d \theta
$$

where $F(\theta, \tau)$ is the force calculated for the single fiber in section IV and the probability density function $p(\theta)$ satisfies $\int_{0}^{1} p d \theta=1$. For the purposes of clarity we shall first assume that $p=1$, but non-uniform distributions will be discussed in section VI. We now calculate $\bar{F}(t)$ for both friction laws and compare the results with experimental measurements.

\section{A. Constant friction law}

From (23) and (24) we can see that for $0<\theta<\frac{1}{2}$ and $\tau<\frac{1}{\alpha}$

$$
F(\theta, \tau)= \begin{cases}0 & \text { if } 0<\theta<\frac{\alpha \tau}{1+\alpha} \\ \left(1+\frac{1}{\alpha}\right) \theta-\tau & \text { if } \frac{\alpha \tau}{1+\alpha}<\theta<\frac{\alpha \tau}{2} \\ \frac{\alpha-1}{2} \tau & \text { if } \frac{\alpha \tau}{2}<\theta<\frac{1}{2}\end{cases}
$$

whereas for $\frac{1}{\alpha}<\tau<\frac{\alpha+1}{2 \alpha}$

$$
F(\theta, \tau)=\left\{\begin{array}{ll}
0 & \text { if } 0<\theta<\frac{\alpha \tau}{1+\alpha} \\
\left(1+\frac{1}{\alpha}\right) \theta-\tau & \text { if } \frac{\alpha \tau}{1+\alpha}<\theta<\frac{1}{2}
\end{array} .\right.
$$

The function $F(\theta, \tau)$ will be symmetric about $\theta=\frac{1}{2}$ and so from (30) with $p=1$,

$\frac{\bar{F}(t)}{T_{s} N}= \begin{cases}\frac{(\alpha-1) U t}{2 l}\left(1-\frac{\alpha(\alpha+3) U t}{2 \ell(\alpha+1)}\right) & 0<t<\frac{l}{U \alpha} \\ \frac{(\alpha+1)}{4 \alpha}\left(1-\frac{2 \alpha U t}{(1+\alpha) \ell}\right)^{2} & \frac{l}{U \alpha}<t<\frac{l(1+\alpha)}{2 \alpha U}\end{cases}$

as illustrated in Figure 12. 


\section{B. Dynamic friction law}

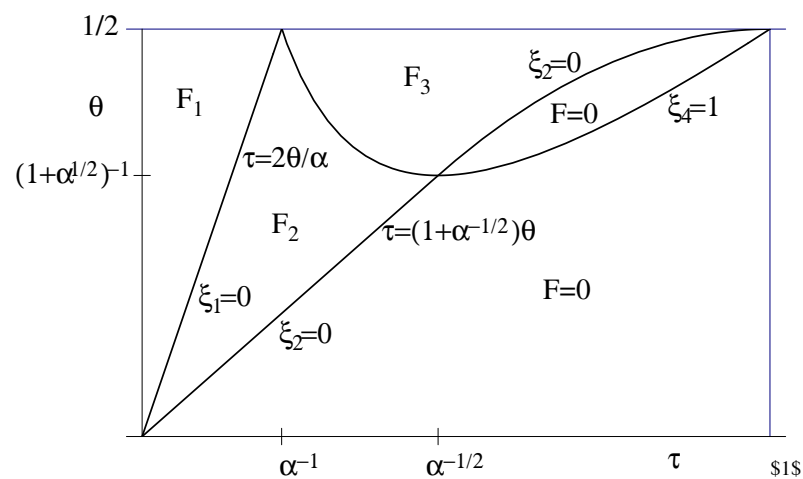

FIG. 13: A diagram of the regions in the $(\theta, \tau)$ plane where $F$ is given by $(34)-(36)$ when a dynamic friction law is applied.

The solutions for $F(\theta, \tau)$ given by (27) - (29) are given below and the regions of validity are illustrated in the $(\theta, \tau)$ plane in Figure 13. Here

$$
\begin{aligned}
F_{1} & =\frac{\alpha-1}{4} \tau \text { for } \quad \frac{\alpha \tau}{2}<\theta<\frac{1}{2} \\
F_{2} & =\frac{(\alpha-1)(\alpha-2)(\theta-\tau)}{\alpha^{2}}-\frac{(\alpha-1)^{\frac{3}{2}}\left(\theta^{2}-4 \theta \tau+2 \tau^{2}\right)}{\alpha^{2} \sqrt{\tau(2 \theta-\tau)}} \\
& \text { if } \frac{\sqrt{\alpha}}{1+\sqrt{\alpha}} \tau<\theta<\min \left\{\frac{\alpha \tau}{2}, \frac{\alpha \tau^{2}-2 \tau+1}{2 \tau}\right\} \\
F_{3} & =\frac{1}{4(1-\tau)^{3}}\left[(1-\tau)^{4}-\left(\frac{\alpha-1}{\alpha}(2 \theta-1)\right)^{2}\right] \\
& \text { if } \max \left\{\frac{1}{2}-\frac{\alpha(1-\tau)^{2}}{2(\alpha-1)}, \frac{\alpha \tau^{2}-2 \tau+1}{2 \tau}\right\}<\theta<\frac{1}{2}(36)
\end{aligned}
$$

and $F$ is zero for other values of $\theta$ in $\left(0, \frac{1}{2}\right)$. Once again $F$ is symmetric about $\theta=\frac{1}{2}$ and so

$$
\bar{F}(\tau)=2 T_{s} N \int_{0}^{\frac{1}{2}} F(\theta, \tau) p(\theta) d \theta .
$$

Evaluating this integral (37) with $p=1$ leads to a complicated expression for $\bar{F}$ which is plotted in Figure 14 for $\alpha=2$.

\section{COMPARISONS WITH EXPERIMENT}

The functions shown in Figures 12 and 14 can now be compared with the experimental results of Dr Mahmoudi which are shown in Figure 2. He performed a series of experiments in which a tuft of fibers is clamped at each end and then the two clamps are moved apart at constant speed $U$. In these experiments he considered three different types of fiber and varied both the speed $U$ and the initial distance between the clamps d. In our model

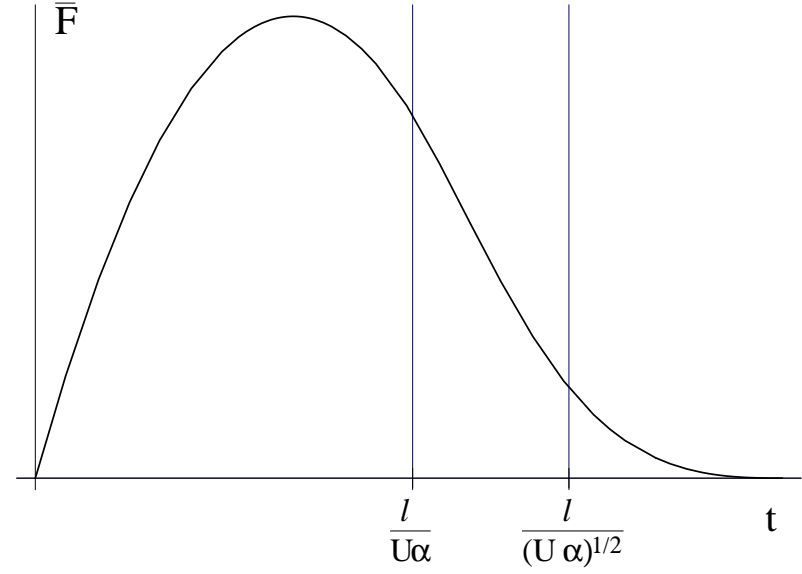

FIG. 14: The averaged force $\bar{F}$ from $(34)-(36)$ and (37) when $\alpha=2$.

we have assumed that only the fibers that cross the plane midway between the two clamps can move and that all the other fibers in the tuft will stay fixed relative to the clamps. This assumption is more likely to hold if the distance between the clamps is small.

The first thing to notice from the experiments is that, as shown in Figure 2, the magnitude of the force depends on the velocity $U$. From (33) and the fact that $T_{s}=\hat{f} \ell$ we see immediately that this is not true for the constant friction law whereas for the dynamic friction law, where $T_{s}=U \lambda \ell$, we predict that the force will depend linearly on $U$ as shown in Figure 14. Although the linear dependence on velocity is only approximately seen in Figure 2, it is well within the bounds of experimental error. The experiments are extremely difficult to perform since no two tufts are exactly the same size and the forces being measured are very small. Nevertheless the evidence of these experiments certainly gives weight to the idea of a velocity-dependent friction law.

We have plotted three tuft breaking forces in Figure 15 where the interconnecting tuft fibers have lengths in either tuft that are (i) uniformly distributed, (ii) more likely to be distributed with the same length in either tuft and (iii) more likely to be distributed with a short length in one tuft and remainder in the other. The latter of these three possible forms for $p$ is in better agreement with experiment. Our model is applicable when a tuft fractures into two independent tufts with interconnecting fibers. The fracture surface will be the region in the tuft where there is least resistance to the applied stress, and this will depend on the number of contact points and the entanglement. In a region where there are a large number of fiber midpoints, for fibers that are uniformly entangled, the fibers are symmetrically embedded in the tuft and would produce greater resistance than a region where there are lots of end points which are only embedded on one side of the fiber. This suggests that the fracture will occur along a surface that is in the vicinity of 

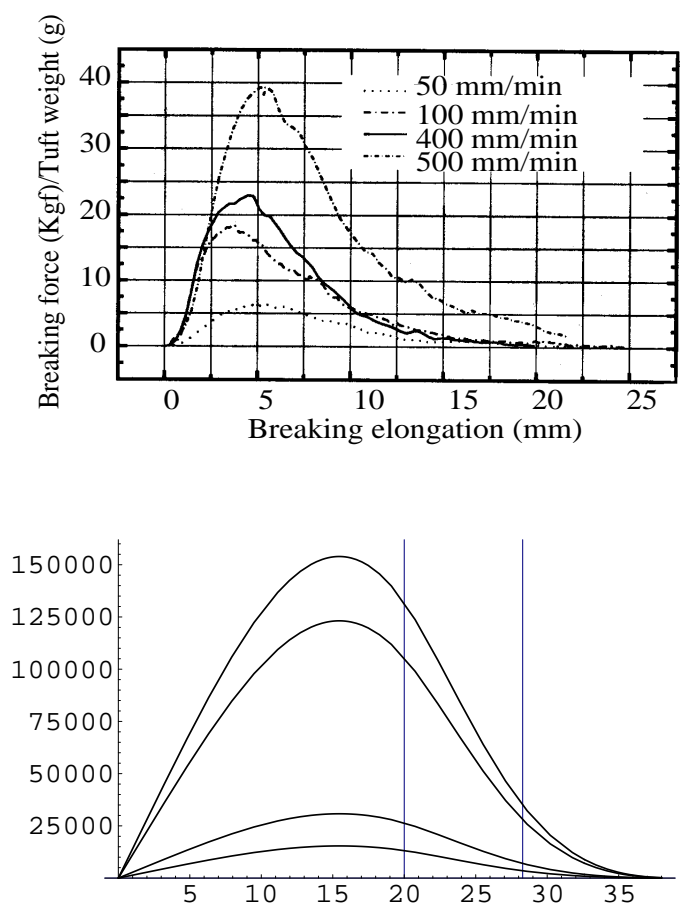

(ii) $\mathrm{p}(\theta)=30 \theta^{2}(\theta-1)^{2}$

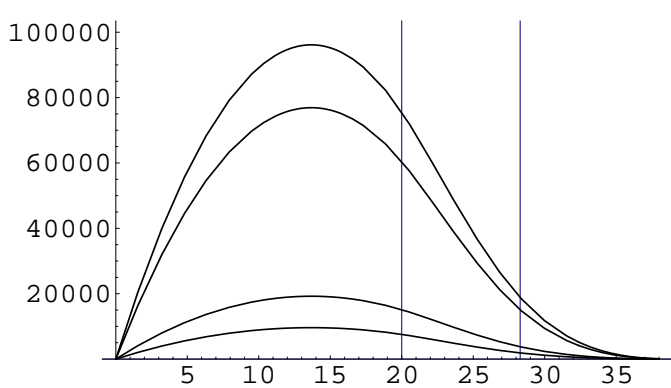

(i) $\mathrm{p}(\theta)=1$

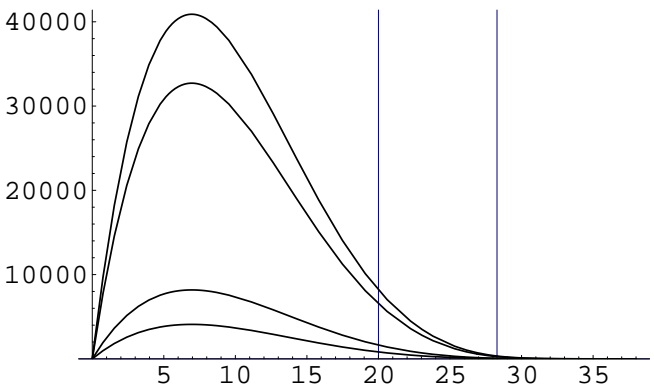

(iii) $\mathrm{p}(\theta)=12(\theta-1 / 2)^{2}$

FIG. 15: $\bar{F}$ plotted against extension, $U t$, for $U=5,10,40,50 \times \frac{10^{-3}}{6} \mathrm{~m} / \mathrm{s}$ and $\ell=0.04 \mathrm{~m}$ using formula (37) for dynamic friction.

more fiber end-points. Hence we suggest that $p(\theta)$ should be larger close to 0 or 1 at the structurally weakest cross section of the tuft, and this supports the experimental comparison in Figure 15 (iii).

\section{CONCLUSIONS}

We considered the withdrawal of a single fiber from a dense and entangled tuft, where the friction at contact points with neighbouring fibers were the dominant force that opposed motion. We introduced the concept of "effective length", the length over which friction forces act, and suggest how its evolution relates to entanglement, initial fiber slackness and the tortuosity of withdrawal path. Two constituitive laws for dynamic friction were considered. The resulting withdrawal forces in Figure 6 captured the essential qualitative features of the experimental data in Figure 1, and we were able to quantify the two model parameters.

In order to develop a model for tuft breaking, we began by considering the behaviour of a single fiber, embedded in adjacent tufts that were being pulled apart. Then, we presented a model for tuft breaking, by considering the case of a tuft that fractures and divides into two tufts, held together by many interconnecting fibers. To allow for variations in the ratio of initial fiber lengths embedded in adjacent tufts, a probability density function was introduced, but its explicit form would depend on the location of fracture. It can be seen in Figure 15 that this function affects how skewed the forces resisting the breaking motion are over time. The results indicate that fracture will occur at a cross-section where the density of fiber end-points is highest, and so most of the interconnecting fibers are not evenly embedded on either side of the eventual fracture.

\section{Acknowledgments}

We wish to express our gratitude to the EPSRC (grant number GR/L62153) and Crosrol Ltd. who funded the interdisciplinary project through which this research was undertaken. We are also grateful to Prof C. Lawrence, Dr. Abbas, Mr. B. Greenwood, Dr. C. Iype and Dr. M. Mahmoudi of the Department of Textile Industries at Leeds University for many useful discussions. This work was also supported by the Fonds National de la Recherche Scientifique (FNRS, Belgium).
[1] G. K. Batchelor, Journal of Fluid Mechanics 44, 419 (1970).
[2] E. J. Hinch and L. G. Leal, Journal of Fluid Mechanics 
71, 481 (1975)

[3] R. R. Sundararajakumar and D. L. Koch, Journal of NonNewtonian Fluid Mechanics 73, 205 (1997).

[4] A. J. M. Spencer, Deformations of fibre-reinforced Materials, Oxford Science Research Papers (Oxford University Press, 1972).

[5] S. L. Phoenix and I. J. Beyerlein, in Comprehensive Composite Materials, edited by A. Kelly and C. Zweben (Pergamon Press, 2000), vol. 1, chap. 1.20.

[6] M. Doi and S. F. Edwards, The Theory of Polymer Dynamics (Oxford University Press, 1986).

[7] P. G. de Gennes, The Physics of Liquid Crystals (Oxford University Press, 1974).

[8] R. Postle, G. A. Carnaby, and S. De Jong, The mechanics of wool structures (Ellis Horwood Ltd, 1988).

[9] C. Servais, J.-A. E. Manson, and S. Toll, Journal of Rheology 43, 991 (1999).

[10] S. Toll and J.-A. E. Månson, Journal of Rheology 38, 985 (1994).

[11] G. A. Carnaby and G. R. Burling-Claridge, Textile Re- search Journal 66, 90 (1996).

[12] J. W. S. Hearle, in Structural Mechanics of Fibers, Yarns and Fabrics, edited by J. W. S. Hearle, P. Grosberg, and S. Backer (Wiley Interscience, 1969), vol. 1, chap. 6.

[13] A. H. Wilkins, Proceedings of the Royal Society A 462, 517 (2006).

[14] M. E. M. Lee and H. Ockendon, European Journal of Applied Mathematics 16, 145 (2005).

[15] M. R. Mahmoudi, C. A. Lawrence, A. A. Dehghani, B. Greenwood, and C. Iype, Journal of the Textile Institute 93, 197 (2004).

[16] M. R. Mahmoudi, C. A. Lawrence, A. A. Dehghani, and B. Greenwood, Journal of the Textile Institute 95, 147 (2004).

[17] J. W. S. Hearle and A. H. Wilkins, Mechanistic modelling of piling: Part $i$ - detailing of the mechanisms, pre-print (2004).

[18] W. Cooke, Ph.D. thesis, University of Oxford (2000).

[19] M. E. M. Lee, Ph.D. thesis, University of Oxford (2001). 